\title{
Effect of Tobbaco Smoking Active and Passive onThe Kidney Functions
}

\section{Osama Saad Hafez Ahmed ${ }^{1, *}$ MSc, Safwat Farrag Ahmed Ahmed ${ }^{1}$ MD, Hazem Sayed Ahmed Ayoub ${ }^{1}$ MD, Medhat Ali Salah AbdElghafar ${ }^{2}$ MD}

*Corresponding Author:

Osama Saad Hafez Ahmed odama20000saad@gmail.com

Received for publication April 29, 2020; Accepted October 16, 2020; Published online October 16, 2020.

Copyright 2020 The Authors published by Al-Azhar University, Faculty of Medicine, Cairo, Egypt. All rights reserved. This an openaccess article distributed under the legal terms, where it is permissible to download and share the work provided it is properly cited. The work cannot be changed in anyway or used commercially.

doi: 10.21608 /aimj.2020.28894.1212

${ }^{1}$ Internal Medicine Department, Faculty of Medicine, Al-Azhar University, Caira, Egypt.

${ }^{2}$ Clinical Pathology Department, Faculty of Medicine, Al-Azhar University, Caira, Egypt.

\begin{abstract}
Background: Cigarette smoking was listed as one of the most important risk factors for incident chronic kidney disease (CKD). In the general adult population, cigarette smoking has been associated with elevated risk of incident CKD / end stage kidney disease (CKD). This correlation was independent of proven CKD risk factors including age, hypertension, DM and BMI.

Aim of work: To compare between tobacco smoking active and passive effect on the kidney functions in a cross section observational study.

Patient and Methods: One Hundred fifty (age and sex matched) patients will be enrolled in a cross section study. The study will be conducted in Nephrology Unit Al-Hussein University Hospital and Air Forces General Hospital.

Results: Comparative study between the 3 groups revealed. Spearman's correlation analysis shows that; SBP, DBP, HR and smoking index, had significant negative correlation with eGFR; with significant statistical difference ( $p<0.01$ respectively). By using ROC-curve analysis, Smoking index at a cutoff point $(>291)$ predicted patients with CKD, with good (83\%) accuracy, sensitivity= 73\% and specificity= $100 \%(\mathrm{p}<$ 0.01).

Conclusion: Our research has shown that smoking, especially heavy smoking (> 30 pack / year), is a significant risk factor for CKD development. For CKD, the correlation was graded as diabetic nephropathy and hypertension the strongest. This findings increase the importance of cessation of smoking in order to minimize the occurrence of CKD and other preventable diseases such as COPD, coronary artery diseases, and cancers.
\end{abstract}

Keywords: Chronic kidney disease; End stage renal disease;chronic vascular disease; Body mass index; Diabetes mellitus.

Disclosure: The authors have no financial interest to declare in relation to the content of this article. The Article Processing Charge was paid for by the authors.

Authorship: All authors have a substantial contribution to the article.

\section{INTRODUCTION}

Cigarette smoking was listed as one of the most important risk factors for incident chronic kidney disease (CKD). In the general adult population, cigarette smoking has been associated with elevated risk of incident CKD / end stage renal disease (CKD). This correlation was independent of proven CKD risk factors including age, hypertension, DM and BMI.1Cessation of smoking substantially reduced the risk of a CKD occurrence, while increased the risk of $\mathrm{CKD}$ for those who quit smoking lasts for several years.Furthermore; smoking is linked with albuminuria / proteinuria cases in the general adult population.2Research in vivo have indicated which nicotine can stimulate the spread of mesangial cells and raise extracellular matrix production. Second, new studies has shown that cigarette smoke contains glycotoxins; that can quickly induce the formation of advanced glycation end products (AGEPs) in vivo and in vitro AGEPs has been shown to raise vascular permeability and encourage pathological vascular changes in renal disease.3The drastic rise in CKD patients worldwide is encouraging nephrologists to adopt preventive strategies. Smoking appears as an significant modifiable kidney risk factor based on several studies documenting a strong correlation between smoking and kidney harm in general population individuals, some research documenting a beneficial impact of cessation of smoking on kidney renal outcome; Numerous reports recording smoking-related modifications that have been shown to be detrimental to the kidney (e.g. an increase in BP) and experimental evidence suggesting that cigarette smoke influences mediator mechanisms known to be involved in the genesis of progressive kidney damage, both invivo and invitro. ${ }^{2}$

Secondhand smoke exposure was linked to higher CKD prevalence as well as development of CKD incident, the prevalence of exposure to passive smoking between the non-smokers were surveyed and the correlation with CKD development in persons who have normal eGFR was investigated.4The high exposure to tobacco among kidney patients (current, passive or past) may be associated with the growing global trend of end-stage renal disease and the gender gap found in that disease. ${ }^{1}$ 


\section{PATIENT AND MATERIALS}

One Hundred fifty (age and sex matched) patient will be enrolled in a cross section study. The study will be conducted in Nephrology Unit Al-Hussein University Hospital and Air Forces General Hospital.

The 150 patient are divided into 3 groups: Group (A): 50 patient heavy smoking. Group(b): 50 patient passive smoking. Group (c): 50 patient normal controls non-smoking.

Inclusion criteria: Patientagingthirty years or more. All patient smoking index (more than three hundred). Patient who exposed to passive smoking more than 5 years.

Exclusion criteria: Age <thirty years smoking, Patient who have malignant diseases. Patient with hepatic impairment.DM, HTN or hyperlipidemia.Obesity and x syndrome.

At enrollment, all patients will be subjected to the following: Full history taking from patients. Complete clinical examination. Basal laboratory work-up: (serum creatinine, Blood Urea, Bun, S. Na, S. K, S. Albumin, CBC, A/C ratio). Glomerular filtration rate (estimated).

This was a case control study conducted on one hundredpatients with chronic kidney disease (CKD) and fifty healthy controls, to compare between tobacco smoking active and passive effect on the kidney functions in a cross section observational study.

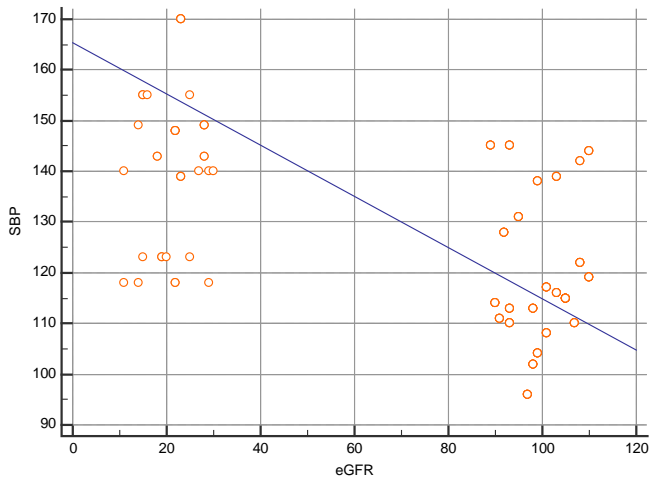

Fig 1: Correlation between eGFR and SBP.

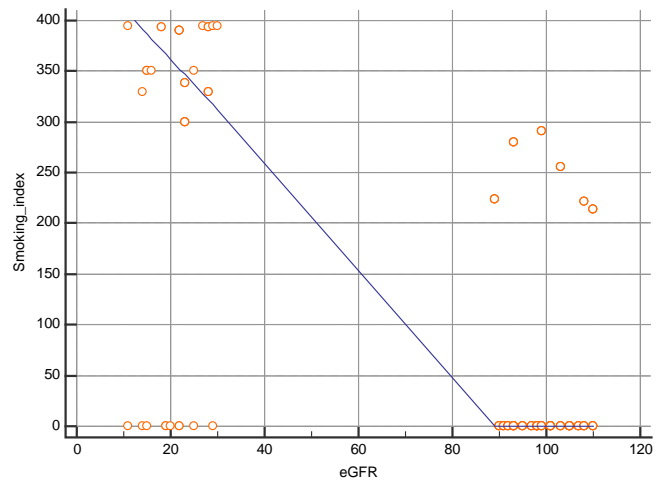

Fig 2: Correlation between eGFR and smoking index.

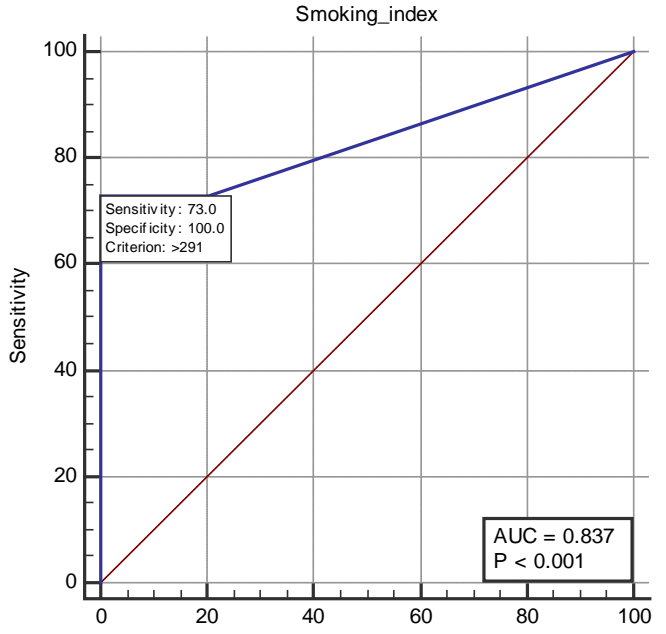

Fig 3: ROC curve of smoking index (CKD).

\section{RESULTS}

comparative analysis revealed between the 3 groups; highly substantial increase in CKD incidence in heavy and passive smokers; relative to control group; with highly significant statistical difference $(\mathrm{p}<0.01)$. Regarding outcome data, (24.7\%) of patients had CKD.

\begin{tabular}{|c|c|}
\hline Variables & Frequency (\%) \\
\hline CKD incidence & $37(24.7 \%)$ \\
\hline
\end{tabular}

Table 1: Outcome data among 150 subjects:Regarding outcome data, (24.7\%) of patients had CKD.

Comparative analysis revealed between the 3 groups; highly substantial increase in K and ACR in heavy smokers; relative to other groups; with highly substantial statistical difference ( $\mathrm{p}<0.01$ respectively).

Comparative analysis revealed between the 3 groups; highly substantial decrease in eGFR in heavy smokers; relative to other groups; with highly substantial statistical difference $(\mathrm{p}<0.01)$.

\section{DISCUSSION}

Regarding clinical data, we found that; the mean age of all patients was $(43.87 \pm 7.1)$ years. Regarding gender of the patients, the majority $(80.7 \%)$ of patients were males; while $(19.3 \%)$ were females, which came in agreement with. ${ }^{5,6,3,7,8}$ and.$^{9}$

Nogueira et al. ${ }^{8}$,reported that, there were 668 nonsmokers and 329 smokers, of whom 233 were exsmokers, and 96 were current smokers, with mean age $46.2 \pm 13.9$.

Gomboset al. ${ }^{9}$ recorded that while 102 patients (25 $\%$ ) continued to smoke (S group). 300 (75\%) were non-Smokers (NS group). Patients in group $\mathrm{S}$ smoked $12.4 \pm 12.4$ pack-months (ppm); their CO was high in exhalation $(9.1 \pm 6.8 \mathrm{ppm})$.

Dülgeret al. ${ }^{5}$,reported that, the topics covered in the study were categorized into 3 groups: Group I (active 
smokers; $n=24)$ : This group composed of participants who smoked on average 20 cigarettes a day, $33.46 \pm 6.73$ years of age. Group II (passive smokers; $n=20$ ): This group composed of participants who were not smoking but subjected to cigarette smoking due to the active smokers who stayed with them during the day for a minimum of 5 6 hours, at age $33.35 \pm 9.53$. Group III (control group; $n=20$ ): This group composed of healthy participants with age $27.55 \pm 8.59$ who did not smoke and did not meet smokers.

Dülger et al. ${ }^{5}$, reported that, the number of participants with a urinary microalbumin / creatinine ratio below or above $30 \mathrm{mg} / \mathrm{g}$, that is the therapeutic limit for confirming the existence of microalbuminuria, was calculated.In 3 of 24 active smokers the rate was above $30 \mathrm{mg} / \mathrm{g}$ and in 2 of 20 passive smokers. In all subjects in the control group the rate was below $30 \mathrm{mg} / \mathrm{g}$.

Dülgeret al. ${ }^{5}$, reported that, in active smokers, the levels of urine microalbumin increased relative to passive smokers and controls. This difference was statistically significant as compared with the control group ( $\mathrm{p}<0.01)$. The urine microalbumin / creatinine ratio was significantly higher in both passive and active smokers relative to the control group $(p<0.01)$.

Mouhamed et al. ${ }^{6}$, reported that, The initial research was performed on 300 volunteer participants, of which 138 non-smokers ( 76 women and 62 men) were $35,6 \pm 16,0$ years old and 162 current smokers ( 17 women and 145 men ) were 38,0 $\pm 17,5$ years old.

Mouhamed et al. ${ }^{6}$, reported that, in this research, we observed that,in smokers plasma creatinine levels decreased significantly relative to non-smokers, while those are not pathologic values. This will show that all of the participants examined are without renal failure.

Peraza et al. ${ }^{3}$, reported that, 664 individuals without exclusions were investigated. The participation was $73 \%$ : women $77 \%$ and men $66 \%$.The highest was $86 \%$ (men in the sugarcane community at high altitude) and the lowest response was $49 \%$ (men in the coffee community)

We found that; the mean BMI of all patients was $(23.98 \pm 3.9)$; while the mean systolic and diastolic BPs were $(124.8 \pm 17.2),(86.5 \pm 6.9) \mathrm{mmHg}$ respectively, which came in agreement with. ${ }^{3,5,6,7,8}$ and. ${ }^{10}$

Yacoubet al. ${ }^{4}$,recorded that, smoking was substantially correlated with CKD risk due to diabetic nephropathy $(\mathrm{OR}=2.24$, CI 95\% 1.27-3.96, $\mathrm{p}=0.005)$ and hypertension (OR $=2.85$, CI $95 \%$ 1.27-6.39, $\mathrm{p}=0.01$ ).

10 reported that, in the sub-sample of subjects with available body composition data $(\mathrm{N}=3780)$, lean body mass showed similar findings, with an average decline of 20.9 (95 \% CI: 21.8 to 20.0) $\mathrm{mL} /$ minute per $1.73 \mathrm{~m} 2$ for IQR increases in serum cotinine concentrations.

García-Esquinas et al. ${ }^{10}$,reported that, present smokers were also more likely than ever to have albuminuria, and subjects with hypertension were at elevated risk of albuminuria when subjected to secondhand smoke.20 There is, however, inconclusive evidence about second-hand smoke and CKD.No population-based studies have assessed the correlation between tobacco and renal function in children.

Comparative analysis revealed between the 3 groups; highly substantial increase in hemoglobin, platelets, creatinine, urea, BUN, urinary albumin, in heavy and passive smokers; compared to control group; with highly substantial statistical difference $(\mathrm{p}<0.01$ respectively), which came in agreement with. ${ }^{3,4,5,6 \text {, }}$ 10,11

Arain et al. $^{11}$, reported that, Mean creatinine clearance of exposed kidney patients (EKPs) in urine samples was substantially reduced relative to referent-diseased patients $(\mathrm{p}<0.01)$ andcollected in acid-washed, decontaminated polyethylene tubes as morning urine samples (spot test) (Kartell1, Milan, Italy).

Comparative analysis revealed between the 3 groups; highly significant increase in K and ACR in heavy smokers; compared to other groups; with substantial statistical difference $(\mathrm{p}<0.01$ respectively), which came in agreement with. ${ }^{4,5,10,12}$.

Yacoubet al. ${ }^{4}$,reported that, urinary albumin is well known to be a reliable marker of glomerular injury, and the fact that smoking is linked to albuminuria indicates direct or indirect smoking-induced renal damage. Participants with critical hypertension noticed that in smokers, the rate of microalbuminuria was almost twice that in non-smokers. Many studies have found similar findings.

Obertet al. ${ }^{12}$,reported that, to decide whether mesangial cell expansion induced by ETS was correlated withalterations in urinary protein excretion, we tested albuminuria diabetic mice that had been exposed to air and ETS.In db / dbnephropathic mice, diabetic mice exposed to air had urine albumin excretions within the range reported by others 10; however, contrary to our Western blot analysis and morphometric analysis, exposure to ETS has not substantially altered albumin urinary excretion: control $(1.03 \pm 0.16 \mathrm{mg}$ albumin/mg creatinine) versus ETS $(1.04 \pm 0.13 \mathrm{mg}$ albumin/mg creatinine; Pvalue not significant).

Comparative analysis revealed between the 3 groups; highly significant decrease in eGFR in heavy smokers; relative to other groups; with highly substantial statistical difference ( $p<0.01$ ), which came in agreement with. ${ }^{4,10}$ and ${ }^{12}$.

Yacoub et al. ${ }^{4}$,reported that, GFR was higher in smoking patients by a factor of 1.44 relative to insulin-dependent diabetes mellitus (IDDM) nonsmoking patients and by a factor of 1.66 between NIDDM patient.

Comparative analysis revealed between the 3 groups; highly substantial increase in CKD incidence in heavy and passive smokers; relative to control group; with highly substantial statistical difference $(\mathrm{p}<0.01)$, which came in agreement with. ${ }^{3}$ and ${ }^{4}$ 
Yacoubet al. ${ }^{4}$, reported that,chronic kidney disease (CKD) prevalent major health issue which has led to the need renal substitute treatment increasing. The causes and effects of cardiovascular risk factors such as diabetes, obesity and hypertension are related to CKD. Usage of smoking and phenacetine is further established risk factors which lastly lead to CKD.

Peraza et al. ${ }^{3}$, reported that, smoking is also responsible for the worsening of lupus nephritis renal function , in a retrospective study of 160 adults, smoking was found to be an independent risk factor for the faster development of CKD during nephritis. As urinary albumin is a reliable predictor of glomerular injury, it is possible that the association between smoking and albuminuria suggests direct or indirect renal damage caused by the smoking. It is responsible for worsening kidney function by growing the risk of microalbuminuria, increasing the development from microalbuminuria to proteinuria and resulting in diabetic nephropathy that contributes to renal disease (CKD) in the end stage.

Multiple regression analysis shows that; after applying (Forward method) and entering some predictor variables; the increase in BMI and the decrease in smoking index; had an independent effect on increasing eGFR; with significant statistical difference ( $p<0.05$ respectively), which came in agreement with ${ }^{4}$

Yacoubet al. ${ }^{4}$,reported that, urinary albumin is well known to be a reliable marker of glomerular injury, and the fact that smoking is linked to albuminuria suggests direct or indirect smoking-induced renal damage. Participants with critical hypertension and noticed that the incidence of microalbuminuria in smokers was nearly double that in non-smokers. Many studies have found similar findings.

By using ROC-curve analysis, Smoking index at a cutoff point (>291) predicted patients with CKD; with good massive effect on the kidney functions in a cross section observational study; which came in agreement with. ${ }^{13}$

\section{CONCLUSION}

Our research has shown that smoking, especially heavy smoking (> 30 pack / year), is a significant risk factor for CKD development. For CKD, the correlation was graded as hypertension and diabetic nephropathy the strongest. These findings increase the importance of cessation of smoking in order to minimize CKD and other preventable disorders such as coronary artery diseases; COPD; and cancers.

\section{REFERENCES}

1. Xia J, Wang L, Ma Z, Zhong L, Wang Y, Gao Y, He L, Su X (2017): Cigarette smoking and chronic kidney disease in the general population: a systematic review and meta-analysis of prospective cohort studies. Nephrology Dialysis Transplantation. 32(3):475-87.

2. Barbato A, D’Elia L, Perna L, Molisso A, Iacone R, Strazzullo P, Galletti F (2019): Increased Microalbuminuria Risk in Male Cigarette
Smokers: Results from the "Olivetti Heart Study" after 8 Years Follow-Up. Kidney and Blood Pressure Research. 44(1):33-42.

3. Peraza S, Wesseling C, Aragon A, Leiva R, García-Trabanino RA, Torres C, Jakobsson K, Elinder CG, Hogstedt C (2012): Decreased kidney function among agricultural workers in El Salvador. American Journal of Kidney Diseases. 59(4):531-40.

4. Yacoub R, Habib H, Lahdo A, Al Ali R, Varjabedian L, Atalla G, Akl NK, Aldakheel S, Alahdab S, Albitar S (2010): Association between smoking and chronic kidney disease: a case control study. BMC public health. 10(1):731.

5. Dülger H, Dönder A, Şekeroğlu MR, Erkoç R, Özbay B (2011): Investigation of the relationship between serum levels of cotinine and the renal function in active and passive smokers. Renal failure. 33(5):475-9.

6. Mouhamed DH, Ezzaher A, Neffati F, Douki W, Gaha L, Najjar MF (2011): Effect of cigarette smoking on plasma uric acid concentrations. Environmental health and preventive medicine. 16(5):307-12.

7. Cumberbatch MG, Rota M, Catto JW, La Vecchia C (2016): The role of tobacco smoke in bladder and kidney carcinogenesis: a comparison of exposures and meta-analysis of incidence and mortality risks. European urology. 70(3):458-66.

8. Nogueira JM, Haririan A, Jacobs SC, Cooper M, Weir MR (2010): Cigarette smoking, kidney function, and mortality after live donor kidney transplant. American journal of kidney diseases. 55(5):907-15.

9. Gombos P, Langer RM, Korbely R, Varga M, Kaposi A, Dinya E, Müller V (2010): Smoking following renal transplantation in Hungary and its possible deleterious effect on renal graft function. InTransplantation proceedings. 42(6)L: 2357-9.

10. García-Esquinas E, Loeffler LF, Weaver VM, Fadrowski JJ, Navas-Acien A (2013): Kidney function and tobacco smoke exposure in US adolescents. Pediatrics. 131(5):1415-23.

11. Arain MB, Kazi TG, Baig JA, Afridi HI, Brehman KD, Panhwar H, Arain SS (2015): Co-exposure of arsenic and cadmium through drinking water and tobacco smoking: risk assessment on kidney dysfunction. Environmental Science and Pollution Research. 22(1):350-7.

12. Obert DM, Hua P, Pilkerton ME, Feng W, Jaimes EA (2011): Environmental tobacco smoke furthers progression of diabetic nephropathy. The American Journal of the Medical Sciences. 341(2):126-30.

13. Panhwar AH, Kazi TG, Afridi HI, Arain SA, Arain MS, Brahaman KD, Arain SS (2016): Correlation of cadmium and aluminum in blood samples of kidney disorder patients with drinking water and tobacco smoking: related health risk. Environmental geochemistry and health. 38(1):265-74. 